\title{
Assessment of Teratogenecity and Embryo Toxicity of Dye Wastewater Untreated Sludge from Sanganer on Swiss Albino Mice when Administered during Growth Period of Gestation
}

\section{Himani* $^{*}$}

Health of Urban Poor, Indian Institute of Health Management Research (IIHMR), Jaipur, India

\begin{abstract}
Water and soil pollution is inherited through the uncontrolled and indiscriminate discharge of pollutants from domestic, commercial and industrial sources into water bodies. This is visible everywhere and the water pollution problem is progressively mounting in our country. The present investigation was carried out to study the toxic effects of non treated sludge leachate from textile and dye industries treated in Sanganer, Rajasthan on lactation, weaning and neonatal growth in mice. Two groups of ten pregnant Swiss albino mice each were given sludge leachate of $0.1 \%$ and $0.01 \%$ dilution with water and libitum from $15^{\text {th }}$ day of gestation till lactation and weaning to cover the critical period of growth. Weight gain of the dams, weight gained by the neonates, sex ratio, viability index, growth index, weaning index were recorded and external malformations were examined.
\end{abstract}

Maternal toxicity due to sludge leachate was evident by significant reduction in body weight gain, substantial loss of body fur and restlessness. Neonatal toxicity was evident by decreased average body weight gain, reduced viability index and weaning index accompanied with retarded growth and vigorous neck movement.

Keywords: Leachate; Neonates; Viability index; Weaning index; Growth index

\section{Introduction}

Dying of clothes has been in vogue for long period and it is practiced as cottage industry in many parts of India. At present, about 10,000 of dyes are known out of which over 5,000 are commercial products. These chemicals have got potential to cause harm to human beings, other living creatures, plants, property and the environment, in general. They may be toxic, highly reactive, explosive or inflammable or may have the combination of these characteristics. The problem of toxic hazards is already reaching alarming proportion in India and is bound to grow with increasing industrialization

In India, most of the liquid wastes are primarily discharged in the aquatic ecosystem often without any pretreatment, which holds true for other developing countries also [1].

Several investigators have reported heavy metal contamination in sewage, effluents and sludge. It is now well known that sewage sludge may contain high concentration of heavy metals [2]. Thus effluent, sludge and other waste materials become a cause of concern. Textile mill effluents have been of major concern in water pollution primarily because of discharge of large qualities of effluents in the nearby water bodies causing health hazards. Textile mill operations consist of weaving, dyeing, printing and finishing. Many processes involve several steps each contributing a particular type of waste.

The above situation can be well depicted in Sanga Nallah (located in Sanganer) that covers a large area of Jaipur. It houses a number of industries (textile and dyeing) that has seen a phenomenal growth during the last two decades. Studies conducted by Mohnot and Dugar [3] and Mohnot and Durve [4] reported toxic chemicals used in dye industries such as dyes, acids and alkalis besides other toxic chemicals like Azo dyes, heavy metals, many of which are known for their hazardous properties.

The toxicity of both untreated and treated textile dye waste water from physicochemical and biological Effluent Treatment Plants (ETP) in the industrial area of Sanganer was assessed by Pratima Soni et al. [5] in order to quantify toxicity reduction.

However, potentially hazardous agents and situations are encountered in this industry, some of which might influence reproductive health. These include solvents, dyes, noise, heat, vibrations, and prolonged standing, heavy metals, etc. [6]. Humans may also be affected from exposure to agents that interfere with ovulation or spermatogenesis [7]. Exposure to such chemical compounds can produce a spectrum of adverse reproductive effects including chromosomal changes, mutations, impotence, sperm abnormalities, early or late fetal loss, still births, decreased birth weights, altered sex ratio, birth defects and childhood malignancies.

In the present study, an attempt has been made to study the effect of sludge leachate at different doses when administered during the growth period (day $15^{\text {th }}$ of gestation till lactation and weaning of pups) in Swiss albino mice.

\section{Materials and Method}

\section{Study area}

The industrial effluent affected area is along the Sanga nallah. In Sanganer town there are many dyeing and printing units, using approximately 10 million litre water per day (mlpd).These industries are generating domestic \& industrial effluent containing high $\mathrm{pH}$,

*Corresponding author: Himani, Water and Sanitation Specialist, Health of Urban Poor, Indian Institute of Health Management Research (IIHMR), Jaipur, India 302001, E-mail: dr.himanitiwari@gmail.com

Received April 25, 2012; Accepted July 17, 2012; Published July 23, 2012

Citation: Himani (2012) Assessment of Teratogenecity and Embryo Toxicity of Dye Wastewater Untreated Sludge from Sanganer on Swiss Albino Mice when Administered during Growth Period of Gestation. J Health Med Inform 3:111. doi:10.4172/2157-7420.1000111

Copyright: (c) 2012 Himani. This is an open-access article distributed under the terms of the Creative Commons Attribution License, which permits unrestricted use, distribution, and reproduction in any medium, provided the original author and source are credited. 
Chemical Oxygen Demand (COD), Biological Oxygen Demand (BOD), Total Dissolved Substance (IDS), Total Suspended Substance (TSS), sulphates \& sodium \& are discharged every day in Sanga nallah.

\section{Test substance}

Test substance used is sludge, collected from drying beds of Sanga nallah. Sludge (untreated) was collected, dried in oven, powdered and then mixed in the ratio of 1:1. It was then diluted by 10 times water and then homogenized. This was then filtered. This filtrate was $100 \%$ leachate, which was then diluted with water according to dose concentration of $1 / 100$ and $1 / 10$ respectively.

\section{Experiment protocol}

$$
0 \text { day }
$$
15 day exposure 21 day lactation 22 day Autopsy

\section{(Birth)}

(Weaning)

Five to six weeks old Swiss albino mice were paired in the ratio of 3 females: 1 male. The females were checked for the presence of the vaginal plug. The day a vaginal plug was seen was taken as day 0 of gestation and the female was presumed to be pregnant. They were caged singly and were assigned randomly to the different dose groups. They were given dose concentration of leachate according to the group 1/10 and $1 / 100$ along with the control group receiving simple tap water. Five inseminated females per test group were treated during the last quarter of pregnancy and through lactation and weaning. The toxicity of leachate was assessed by calculating the gestation index, viability index, weaning index and growth index. The data obtained were statistically evaluated using Mann- Whitney U test [8] and Student's T test.

Viability Index (at day 4 of age)

$=$ No. of alive pups at postnatal day 4/ No. of alive pups born ${ }^{\star} 100$.

Weaning Index

$=$ No. of alive pups at 21 days after birth / No. of pups maintained at 4 days of age ${ }^{\star} 100$.

Growth Index was calculated as average weights of male and female pups at days 4, 7, 14 and 21 days of age (Figure 1).

\section{Experiment with different leachate concentrations}

Treatment during growth period: Two groups of pregnant female mice were exposed to $1 / 100$ and $1 / 10$ concentration of leachate in drinking water along with the control group from day $15^{\text {th }}$ of gestation till lactation and weaning to cover the critical period of growth.

\section{Result and Discussion}

Exposure of leachate with $1 / 100$ and $1 / 10$ concentration in drinking water from day $15^{\text {th }}$ till lactation and weaning revealed reduced maternal weight gain at both the dose levels. The leachate also affected the weight of the pups at the time of birth. Sex ratio was also affected along with the weaning, growth and viability index. The number of alive pups also reduced significantly at higher dose group.

Various parameters were observed to assess the neonatal growth of pups treated with different concentration of sludge leachate. In the present investigation, the untreated sludge leachate from Sanga Nallah at the tested dose levels produced maternal toxicity. The toxic symptoms in mothers ranged from muscular tremors, ataxia, convulsions, hypersalivation, and excessive lacrimation to restlessness and lethargy. Besides toxic symptoms the leachate also caused reduced weight gain by dams during gestation. Both the doses viz.: 1/100 and $1 / 10$ concentration of leachate in drinking water when given during the growth periods of gestation (last quarter of pregnancy) exhibited reduced weight gain by treated dams (Tables 1a and 1b). The high dose was more effective in lowering maternal weight gain. This could probably be associated to lesser number of fetuses obtained from the treated animals. The leachate also affected the weight of the pups at the time of birth (Tables $2 \mathrm{a}, 2 \mathrm{~b}$ and $2 \mathrm{c}$ ). Sex ratio was also affected along with the weaning, growth and viability index (Tables $3 a$ and $3 b)$. The number of alive pups also reduced significantly at higher dose group (Figures 2 and 3).

The neonatal viability in the leachate experiments during peri/

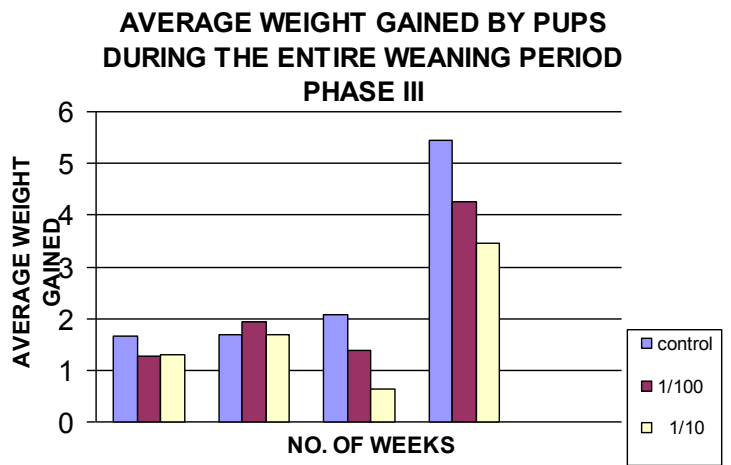

Figure 1: Average weight gained by pups during the entire weaning period phase III.

NUMBER OF PUPS ALIVE : PHASE III

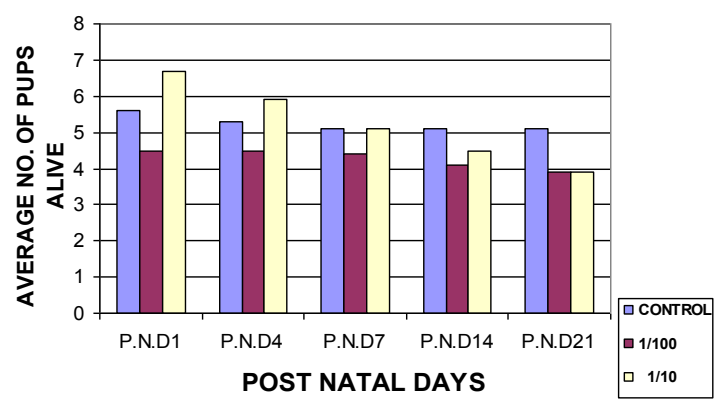

Figure 2: Number of pups alive: phase III.

\section{AVERAGE WEIGHT OF PUPS OF PHASE III}

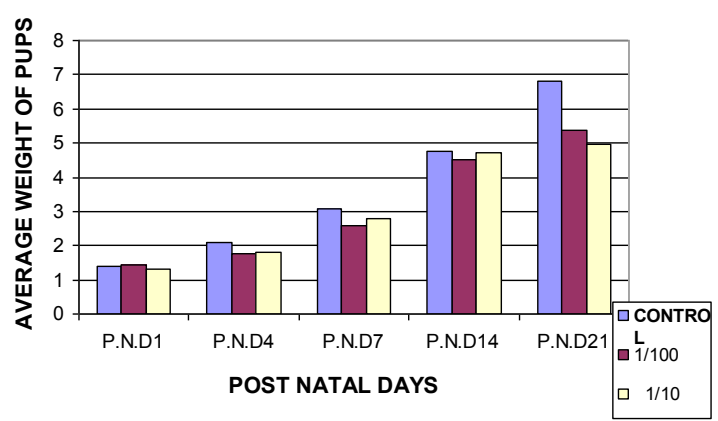

Figure 3: Average weight of pups of phase III. 
Citation: Himani (2012) Assessment of Teratogenecity and Embryo Toxicity of Dye Wastewater Untreated Sludge from Sanganer on Swiss Albino Mice when Administered during Growth Period of Gestation. J Health Med Inform 3:111. doi:10.4172/2157-7420.1000111

Page 3 of 5

\begin{tabular}{|c|c|c|c|c|c|c|c|c|c|c|c|c|}
\hline \multicolumn{5}{|c|}{ CONTROL } & \multicolumn{4}{|c|}{ 1/100 DILUTION OF SLUDGE LEACHATE } & \multicolumn{4}{|c|}{ 1/10 DILUTION OF SLUDGE LEACHATE } \\
\hline S. No. & $1^{\text {st }}$ Week & $2^{\text {nd }}$ Week & $3^{\text {rd }}$ Week & $\begin{array}{l}\text { Complete } \\
\text { Weaning }\end{array}$ & $1^{\text {st }}$ Week & $2^{\text {nd }}$ Week & $3^{\text {rd }}$ Week & $\begin{array}{l}\text { Complete } \\
\text { Weaning }\end{array}$ & $1^{\text {st }}$ Week & $2^{\text {nd }}$ Week & $3^{\text {rd }}$ Week & $\begin{array}{l}\text { Complete } \\
\text { Weaning }\end{array}$ \\
\hline 1 & 1.98 & 2.03 & 2.49 & 6.51 & 1.73 & 2.37 & 1.68 & 5.79 & Died & On & $4^{\text {th }}$ & Day \\
\hline 2 & 1.27 & 1.47 & 1.85 & 4.60 & 1.02 & 2.24 & 0.65 & 3.91 & 0.88 & 2.00 & 1.28 & 4.17 \\
\hline 3 & 0.97 & 0.96 & 1.66 & 3.59 & 0.64 & 3.68 & 1.46 & 5.79 & 1.78 & 1.81 & 0.15 & 3.74 \\
\hline 4 & 2.04 & 2.75 & 2.01 & 6.81 & 2.26 & 1.23 & --.-- & ------ & 2.56 & 3.93 & 1.48 & 7.99 \\
\hline 5 & 1.55 & 1.44 & 1.93 & 4.93 & 1.64 & 1.24 & 3.06 & 5.94 & 0.07 & 1.71 & -0.36 & 1.42 \\
\hline Total & 7.81 & 8.65 & 9.94 & 26.44 & 7.29 & 10.76 & 6.85 & 21.43 & 5.29 & 9.45 & 3.27 & 17.32 \\
\hline Average & $1.56 \pm 0.14$ & $1.73 \pm 0.24$ & $1.99 \pm 0.15$ & $5.29 \pm 0.48$ & $1.46 \pm 0.22$ & $2.152 \pm 0.31$ & $1.37 \pm 0.32$ & $4.28 \pm 0.75$ & $1.05 \pm 0.24$ & $1.89 \pm 0.36$ & $0.654 \pm 0.36$ & $3.46 \pm 0.83$ \\
\hline
\end{tabular}

Student's $t$ test: *Significant difference $(p<0.05)^{\star *}$ highly significant difference $(p<0.01)$

Table 1(a): Effect of leachate on maternal weight gain.

\begin{tabular}{|c|c|c|c|c|c|c|c|c|c|c|c|c|c|c|c|}
\hline \multicolumn{4}{|c|}{ Post Natal Day 1} & \multicolumn{3}{|c|}{ Post Natal Day 4} & \multicolumn{3}{|c|}{ Post Natal Day 7} & \multicolumn{3}{|c|}{ Post Natal Day 14} & \multicolumn{3}{|c|}{ Post Natal Day 21} \\
\hline S.No. & $\begin{array}{l}\text { No. of } \\
\text { pups } \\
\text { alive }\end{array}$ & $\begin{array}{l}\text { Avg. } \\
\text { pup } \\
\text { wgt. }\end{array}$ & $\begin{array}{c}\text { Sex } \\
\text { Ratio } \\
\text { M: F }\end{array}$ & $\begin{array}{l}\text { No. of } \\
\text { pups } \\
\text { alive }\end{array}$ & $\begin{array}{l}\text { Avg. } \\
\text { pup } \\
\text { wgt. }\end{array}$ & $\begin{array}{c}\text { Sex } \\
\text { Ratio } \\
\text { M: F }\end{array}$ & $\begin{array}{l}\text { No. of } \\
\text { pups } \\
\text { alive }\end{array}$ & $\begin{array}{l}\text { Avg. } \\
\text { pup } \\
\text { wgt. }\end{array}$ & $\begin{array}{c}\text { Sex } \\
\text { Ratio } \\
\text { M:F }\end{array}$ & $\begin{array}{c}\text { No. Of } \\
\text { Pups } \\
\text { Alive }\end{array}$ & $\begin{array}{l}\text { Avg. } \\
\text { Pup } \\
\text { Wgt. }\end{array}$ & $\begin{array}{c}\text { Sex } \\
\text { Ratio } \\
\text { M:F }\end{array}$ & $\begin{array}{l}\text { No. of } \\
\text { Pups } \\
\text { Alive }\end{array}$ & $\begin{array}{l}\text { Avg. } \\
\text { Pup } \\
\text { Wgt. }\end{array}$ & $\begin{array}{c}\text { Sex } \\
\text { Ratio } \\
\text { M:F }\end{array}$ \\
\hline 1 & 4 & 1.52 & $2: 2$ & 4 & 2.35 & $2: 2$ & 4 & 3.50 & 2: 2 & 4 & 5.54 & $2 ; 2$ & 4 & 8.04 & $2: 2$ \\
\hline 2 & 5 & 1.91 & $2: 3$ & 5 & 2.30 & $2: 3$ & 5 & 3.19 & $2: 3$ & 5 & 4.66 & $2: 3$ & 5 & 6.52 & $2: 3$ \\
\hline 3 & 6 & 1.44 & $3: 3$ & 5 & 2.18 & $3: 2$ & 5 & 2.41 & $3: 2$ & 5 & 3.37 & $3: 2$ & 5 & 5.03 & $3: 2$ \\
\hline 4 & 5 & 1.18 & $5: 0$ & 5 & 2.19 & $5: 0$ & 5 & 3.22 & $5: 0$ & 5 & 5.97 & $5: 0$ & 5 & 7.99 & $5: 0$ \\
\hline 5 & 8 & 1.12 & $2: 6$ & 6 & 1.34 & $2: 4$ & 5 & 2.68 & $1: 4$ & 5 & 4.12 & $1: 4$ & 5 & 6.06 & $1: 4$ \\
\hline Average & $\begin{array}{c}5.6 \\
\pm 0.40\end{array}$ & $\begin{array}{c}1.43 \\
\pm 0.07\end{array}$ & $\begin{array}{l}50 \\
50\end{array}$ & $\begin{array}{c}5 \\
\pm 0.30\end{array}$ & $\begin{array}{c}2.07 \\
\pm 0.11\end{array}$ & $\begin{array}{l}52.8 \\
47.1\end{array}$ & $\begin{array}{c}4.80 \\
\pm 0.23\end{array}$ & $\begin{array}{c}3.0 \\
\pm 0.14\end{array}$ & $\begin{array}{l}50.98 \\
49.01\end{array}$ & $\begin{array}{c}4.80 \\
\pm 0.23\end{array}$ & $\begin{array}{c}4.53 \\
\pm 0.36\end{array}$ & $\begin{array}{l}50.98 \\
49.01\end{array}$ & $\begin{array}{c}4.80 \\
\pm 0.23\end{array}$ & $\begin{array}{c}6.73 \\
\pm 0.45\end{array}$ & $\begin{array}{l}50.98 \\
49.01\end{array}$ \\
\hline
\end{tabular}

Student's t test: *Significant difference $(p<0.05)^{\star *}$ highly significant difference $(p<0.01)$

Table 1(b): Neonatal observation of mice born to mothers given tap water -control.

\begin{tabular}{|c|c|c|c|c|c|c|c|c|c|c|c|c|c|c|c|}
\hline \multicolumn{4}{|c|}{ Post Natal Day 1} & \multicolumn{3}{|c|}{ Post Natal Day 4} & \multicolumn{3}{|c|}{ Post Natal Day 7} & \multicolumn{3}{|c|}{ Post Natal Day 14} & \multicolumn{3}{|c|}{ Post Natal Day 21} \\
\hline S.No. & $\begin{array}{l}\text { No. of } \\
\text { pups } \\
\text { alive }\end{array}$ & $\begin{array}{l}\text { Avg. } \\
\text { pup } \\
\text { wgt. }\end{array}$ & $\begin{array}{c}\text { Sex } \\
\text { Ratio } \\
\text { M: F }\end{array}$ & $\begin{array}{l}\text { No. of } \\
\text { pups } \\
\text { alive }\end{array}$ & $\begin{array}{l}\text { Avg. } \\
\text { pup } \\
\text { wgt. }\end{array}$ & $\begin{array}{c}\text { Sex } \\
\text { Ratio } \\
\text { M: F }\end{array}$ & $\begin{array}{l}\text { No. of } \\
\text { pups } \\
\text { alive }\end{array}$ & $\begin{array}{l}\text { Avg. } \\
\text { pup } \\
\text { wgt. }\end{array}$ & $\begin{array}{c}\text { Sex } \\
\text { Ratio } \\
\text { M: F }\end{array}$ & $\begin{array}{l}\text { No. of } \\
\text { Pups } \\
\text { Alive }\end{array}$ & $\begin{array}{l}\text { Avg. } \\
\text { Pup } \\
\text { Wgt. }\end{array}$ & $\begin{array}{c}\text { Sex } \\
\text { Ratio } \\
\text { M: F }\end{array}$ & $\begin{array}{l}\text { No. of } \\
\text { Pups } \\
\text { Alive }\end{array}$ & $\begin{array}{l}\text { Avg. } \\
\text { Pup } \\
\text { Wgt. }\end{array}$ & $\begin{array}{c}\text { Sex } \\
\text { Ratio } \\
\text { M: F }\end{array}$ \\
\hline 1 & 4 & 1.52 & $2: 2$ & 4 & 2.35 & $2: 2$ & 4 & 3.50 & 2: 2 & 4 & 5.54 & $2 ; 2$ & 4 & 8.04 & $2 ; 2$ \\
\hline 2 & 5 & 1.91 & $2: 3$ & 5 & 2.30 & $2: 3$ & 5 & 3.19 & $2: 3$ & 5 & 4.66 & $2: 3$ & 5 & 6.52 & $2: 3$ \\
\hline 3 & 6 & 1.44 & $3: 3$ & 5 & 2.18 & $3: 2$ & 5 & 2.41 & $3: 2$ & 5 & 3.37 & $3: 2$ & 5 & 5.03 & $3: 2$ \\
\hline 4 & 5 & 1.18 & $5: 0$ & 5 & 2.19 & 5:0 & 5 & 3.22 & $5: 0$ & 5 & 5.97 & 5:0 & 5 & 7.99 & 5:0 \\
\hline 5 & 8 & 1.12 & $2: 6$ & 6 & 1.34 & $2: 4$ & 5 & 2.68 & $1: 4$ & 5 & 4.12 & $1: 4$ & 5 & 6.06 & $1: 4$ \\
\hline Average & $\begin{array}{c}5.6 \\
\pm 0.40\end{array}$ & $\begin{array}{c}1.43 \\
\pm 0.07\end{array}$ & $\begin{array}{l}50 \\
50\end{array}$ & $\begin{array}{c}5 \\
\pm 0.30\end{array}$ & $\begin{array}{c}2.07 \\
\pm 0.11\end{array}$ & $\begin{array}{l}52.8 \\
47.1\end{array}$ & $\begin{array}{c}4.80 \\
\pm 0.23\end{array}$ & $\begin{array}{c}3.0 \\
\pm 0.14\end{array}$ & $\begin{array}{l}50.98 \\
49.01\end{array}$ & $\begin{array}{c}4.80 \\
\pm 0.23\end{array}$ & $\begin{array}{c}4.53 \\
\pm 0.36\end{array}$ & $\begin{array}{l}50.98 \\
49.01\end{array}$ & $\begin{array}{c}4.80 \\
\pm 0.23\end{array}$ & $\begin{array}{c}6.73 \\
\pm 0.45\end{array}$ & $\begin{array}{l}50.98 \\
49.01\end{array}$ \\
\hline
\end{tabular}

Student's t test: *Significant difference $(p<0.05)^{* *}$ highly significant difference $(p<0.01)$

Table 2 (a): Neonatal observation of mice born to mothers given tap water-control.

\begin{tabular}{|c|c|c|c|c|c|c|c|c|c|c|c|c|c|c|}
\hline Mother No. & $\begin{array}{l}\text { No. of } \\
\text { pups } \\
\text { alive }\end{array}$ & $\begin{array}{l}\text { Av. Pup } \\
\text { Weight. }\end{array}$ & $\begin{array}{c}\text { Sex } \\
\text { Ratio }\end{array}$ & $\begin{array}{l}\text { No. of } \\
\text { pups } \\
\text { alive }\end{array}$ & $\begin{array}{l}\text { Av. Pup } \\
\text { weight }\end{array}$ & $\begin{array}{c}\text { Sex } \\
\text { Ratio }\end{array}$ & $\begin{array}{l}\text { No. of } \\
\text { pups } \\
\text { alive }\end{array}$ & $\begin{array}{l}\text { Av. Pup } \\
\text { weight }\end{array}$ & $\begin{array}{c}\text { Sex } \\
\text { Ratio }\end{array}$ & $\begin{array}{l}\text { No. of } \\
\text { pups } \\
\text { alive }\end{array}$ & $\begin{array}{l}\text { Av. pup } \\
\text { weight }\end{array}$ & Sex Ratio & $\begin{array}{l}\text { No. of } \\
\text { Pups } \\
\text { Alive }\end{array}$ & $\begin{array}{l}\text { Av. Pup } \\
\text { Weight }\end{array}$ \\
\hline 1 & 5 & 1.49 & $1: 4$ & 5 & 2.27 & $1: 4$ & 5 & 3.22 & $1: 4$ & 5 & 5.59 & $1: 4$ & 5 & 7.28 \\
\hline 2 & 7 & 1.44 & $3: 4$ & 7 & 1.61 & $3: 4$ & 7 & 2.46 & $3: 4$ & 6 & 4.71 & $3: 3$ & 6 & 5.36 \\
\hline 3 & 7 & 1.45 & $4: 3$ & 7 & 2.18 & $4: 2$ & 6 & 1.90 & $4: 2$ & 5 & 5.58 & $4: 1$ & 5 & 7.05 \\
\hline 4 & 3 & 1.82 & $2: 1$ & 3 & 2.08 & $2: 1$ & 3 & 4.08 & $2: 1$ & 2 & 5.31 & $1: 1$ & ---- & --.-- \\
\hline 5 & 7 & 1.46 & $5: 2$ & 7 & 2.16 & $5: 2$ & 7 & 3.10 & $5: 2$ & 7 & 4.34 & $5: 2$ & 7 & 7.41 \\
\hline Avg. & $\begin{array}{c}5.8 \\
+0.72\end{array}$ & $\begin{array}{c}1.53 \\
+0.05\end{array}$ & $\begin{array}{l}\mathbf{5 0} \% \\
\mathbf{5 0} \%\end{array}$ & $\begin{array}{c}5.8 \\
\pm 0.70\end{array}$ & $\begin{array}{c}2.04 \\
\pm 0.67\end{array}$ & $\begin{array}{l}52 \% \\
48 . \%\end{array}$ & $\begin{array}{c}5.30 \\
\pm 0.67\end{array}$ & $\begin{array}{c}2.95 \\
\pm 0.35\end{array}$ & $\begin{array}{l}52 \% \\
48 \%\end{array}$ & $\begin{array}{c}5.0 \\
\pm 0.64\end{array}$ & $\begin{array}{c}5.10 \\
\pm 0.52\end{array}$ & $\begin{array}{l}51.2 \% \\
48.7 \%\end{array}$ & $\begin{array}{c}4.60 \\
\pm 0.73\end{array}$ & $\begin{array}{c}5.22 \\
\pm 0.92\end{array}$ \\
\hline
\end{tabular}

Student's t test: *Significant difference $(p<0.05)^{\star *}$ highly significant difference $(p<0.01)$

Table 2(b): Neonatal observation of mice born to mothers given 1/100 concentration of sludge leachate. 
Citation: Himani (2012) Assessment of Teratogenecity and Embryo Toxicity of Dye Wastewater Untreated Sludge from Sanganer on Swiss Albino Mice when Administered during Growth Period of Gestation. J Health Med Inform 3:111. doi:10.4172/2157-7420.1000111

Page 4 of 5

\begin{tabular}{|c|c|c|c|c|c|c|c|c|c|c|c|c|c|c|c|}
\hline \multicolumn{4}{|c|}{ Post Natal Day 1} & \multicolumn{3}{|c|}{ Post Natal Day 4} & \multicolumn{3}{|c|}{ Post Natal Day 7} & \multicolumn{3}{|c|}{ Post Natal Day 14} & \multicolumn{3}{|c|}{ Post Natal Day 21} \\
\hline S. No & $\begin{array}{l}\text { No. of } \\
\text { pups } \\
\text { alive }\end{array}$ & $\begin{array}{c}\text { Avg. pup } \\
\text { wgt. }\end{array}$ & $\begin{array}{c}\text { Sex } \\
\text { Ratio } \\
\text { M: F }\end{array}$ & $\begin{array}{c}\text { No. of } \\
\text { pups alive }\end{array}$ & $\begin{array}{c}\text { Avg. } \\
\text { pup wgt. }\end{array}$ & $\begin{array}{c}\text { Sex Ratio } \\
\text { M: F }\end{array}$ & $\begin{array}{c}\text { No. of } \\
\text { pups alive }\end{array}$ & $\begin{array}{c}\text { Avg. } \\
\text { pup wgt. }\end{array}$ & $\begin{array}{c}\text { Sex } \\
\text { Ratio } \\
\text { M: F }\end{array}$ & $\begin{array}{l}\text { No. of } \\
\text { pups } \\
\text { alive }\end{array}$ & $\begin{array}{c}\text { Avg. } \\
\text { pup wgt. }\end{array}$ & $\begin{array}{c}\text { Sex } \\
\text { Ratio } \\
\text { M: F }\end{array}$ & $\begin{array}{l}\text { No. of } \\
\text { pups } \\
\text { alive }\end{array}$ & $\begin{array}{l}\text { Avg. } \\
\text { pup } \\
\text { wgt. }\end{array}$ & $\begin{array}{c}\text { Sex } \\
\text { Ratio } \\
\text { M: F }\end{array}$ \\
\hline & 5 & 1.06 & $3: 2$ & All died & --- & ----- & ------ & -------- & ------ & ------ & ----- & ------- & ------ & ----- & ------ \\
\hline & 10 & 1.01 & $4: 6$ & 9 & 1.09 & $4: 5$ & 7 & 1.91 & $3: 4$ & 7 & 3.91 & $3: 4$ & 7 & 5.20 & $3: 4$ \\
\hline & 8 & 1.00 & $5: 3$ & 8 & 1.77 & $5: 3$ & 8 & 2.78 & $5: 3$ & 8 & 4.59 & $5: 3$ & 8 & 4.75 & $5: 3$ \\
\hline & 4 & 1.61 & $4: 0$ & 4 & 2.63 & $4: 0$ & 4 & 4.187 & $4: 0$ & 4 & 8.12 & $4: 0$ & 4 & 10.60 & $4: 0$ \\
\hline & 4 & 1.46 & $3: 1$ & 4 & 1.39 & $3: 1$ & 4 & 1.54 & $3: 1$ & 4 & 3.25 & $3: 1$ & 3 & 2.89 & $2: 1$ \\
\hline Average & $\begin{array}{c}6.2 \\
\pm 0.76\end{array}$ & $\begin{array}{r}1.23 \\
\pm 0.07\end{array}$ & $\begin{array}{l}47.76 \% \\
52.23 \%\end{array}$ & $\begin{array}{c}5 \\
\pm 0.76\end{array}$ & $\begin{array}{c}1.38 \\
\pm 0.15\end{array}$ & $\begin{array}{l}62 \% \\
38 \%\end{array}$ & $\begin{array}{c}4.60 \\
\pm 0.76\end{array}$ & $\begin{array}{c}2.08 \\
\pm 0.23\end{array}$ & $\begin{array}{l}60 \% \\
40 \%\end{array}$ & $\begin{array}{c}4.60 \\
\pm 0.76\end{array}$ & $\begin{array}{c}3.97 \\
\pm 0.46\end{array}$ & $\begin{array}{l}60 \% \\
40 \%\end{array}$ & $\begin{array}{c}4.4 \\
\pm 0.46\end{array}$ & $\begin{array}{c}4.68 \\
\pm 0.76\end{array}$ & $\begin{array}{c}56 . \%: \\
44 \%\end{array}$ \\
\hline
\end{tabular}

Student's t test: *Significant difference $(p<0.05)^{\star *}$ highly significant difference $(p<0.01)$

Table 2 (c): Neonatal observation of mice born to mothers given $1 / 10$ concentration of sludge leachate.

\begin{tabular}{|c|c|c|c|c|c|c|c|c|c|c|c|c|c|c|c|}
\hline \multirow[b]{2}{*}{ S. No. } & \multicolumn{5}{|c|}{ CONTROL } & \multicolumn{5}{|c|}{$\begin{array}{c}\text { 1/100 CON OF SLUDGE LEACHATE INDRINKING } \\
\text { WATER }\end{array}$} & \multicolumn{5}{|c|}{$\begin{array}{l}\text { 1/10 CONCENTRATION OF SLUDGE } \\
\text { LEACHATE IN DRINKING WATER }\end{array}$} \\
\hline & 4 & 7 & 14 & 21 & Weanling \% & 4 & 7 & 14 & 21 & Weanling \% & 4 & 7 & 14 & 21 & Weanling \% \\
\hline 1 & 100 & 100 & 100 & 100 & 100 & 100 & 100 & 100 & 100 & 100 & - & - & - & - & \\
\hline 2 & 100 & 100 & 100 & 100 & 100 & 100 & 85.6 & 85.6 & 85.6 & 100 & 70 & 70 & 70 & 70 & 70 \\
\hline 3 & 83.3 & 83.3 & 83.3 & 83.3 & 100 & 85.6 & 71.4 & 71.4 & 71.4 & 100 & 100 & 100 & 100 & 100 & 100 \\
\hline 4 & 100 & 100 & 100 & 100 & 100 & 100 & 66.6 & - & - & 100 & 100 & 100 & 100 & 100 & 100 \\
\hline 5 & 62.5 & 62.5 & 625 & 62.5 & 100 & 100 & 100 & 100 & 100 & 100 & 100 & 100 & 75 & 75 & 75 \\
\hline
\end{tabular}

Table 3 (a): Effect of leachate on Viability and weaning index of pups of all three groups.

\begin{tabular}{|c|c|c|c|c|c|c|c|c|c|c|c|c|c|c|c|}
\hline \multirow[b]{2}{*}{$\begin{array}{l}\text { Mother } \\
\text { No. }\end{array}$} & \multicolumn{5}{|c|}{ CONTROL } & \multicolumn{5}{|c|}{$\begin{array}{l}\text { 1/100 CONCENTRATION OF SLUDGE } \\
\text { LEACHATE }\end{array}$} & \multicolumn{5}{|c|}{$\begin{array}{l}\text { 1/10 CONCENTRATION OF SLUDGE } \\
\text { LEACHATE }\end{array}$} \\
\hline & $\begin{array}{l}\text { Post } \\
\text { Natal } \\
\text { Day } 1\end{array}$ & $\begin{array}{l}\text { Post } \\
\text { Natal } \\
\text { Day } 4\end{array}$ & $\begin{array}{l}\text { Post } \\
\text { Natal } \\
\text { Day } 7\end{array}$ & \begin{tabular}{|c|} 
Post \\
Natal \\
Day 14
\end{tabular} & $\begin{array}{c}\text { Post } \\
\text { Natal } \\
\text { Day } 21\end{array}$ & $\begin{array}{l}\text { Post } \\
\text { Natal } \\
\text { Day } 1\end{array}$ & $\begin{array}{c}\text { Post Natal } \\
\text { Day } 4\end{array}$ & $\begin{array}{l}\text { Post } \\
\text { Natal } \\
\text { Day } 7\end{array}$ & $\begin{array}{c}\text { Post } \\
\text { Natal } \\
\text { Day } 14\end{array}$ & $\begin{array}{c}\text { Post } \\
\text { Natal } \\
\text { Day } 21\end{array}$ & $\begin{array}{l}\text { Post } \\
\text { Natal } \\
\text { Day } 1\end{array}$ & $\begin{array}{l}\text { Post } \\
\text { Natal } \\
\text { Day } 4\end{array}$ & $\begin{array}{l}\text { Post } \\
\text { Natal } \\
\text { Day } 7\end{array}$ & $\begin{array}{c}\text { Post } \\
\text { Natal } \\
\text { Day } 14\end{array}$ & $\begin{array}{c}\text { Post } \\
\text { Natal } \\
\text { Day } 21\end{array}$ \\
\hline & $\begin{array}{l}\text { Avg. pup } \\
\text { wgt. }\end{array}$ & $\begin{array}{l}\text { Avg. } \\
\text { pup } \\
\text { wgt. }\end{array}$ & $\begin{array}{l}\text { Avg. pup } \\
\text { wgt. }\end{array}$ & $\begin{array}{l}\text { Avg. } \\
\text { pup } \\
\text { wgt. }\end{array}$ & $\begin{array}{l}\text { Avg. } \\
\text { pup } \\
\text { wgt. }\end{array}$ & $\begin{array}{l}\text { Avg. pup } \\
\text { wgt. }\end{array}$ & $\begin{array}{l}\text { Avg. pup } \\
\text { wgt. }\end{array}$ & $\begin{array}{l}\text { Avg. Pup } \\
\text { wgt. }\end{array}$ & $\begin{array}{l}\text { Avg. Pup } \\
\text { wgt. }\end{array}$ & $\begin{array}{l}\text { Avg. pup } \\
\text { wgt. }\end{array}$ & $\begin{array}{l}\text { Avg. } \\
\text { pup wgt. }\end{array}$ & $\begin{array}{l}\text { Avg. } \\
\text { pup wgt. }\end{array}$ & $\begin{array}{l}\text { Avg. } \\
\text { pup } \\
\text { wgt. }\end{array}$ & $\begin{array}{l}\text { Avg. } \\
\text { Pup + } \\
\text { wgt. }\end{array}$ & $\begin{array}{c}\text { Avg. } \\
\text { pup wgt. }\end{array}$ \\
\hline 1 & 1.52 & 2.35 & 3.50 & 5.54 & 8.04 & 1.49 & 2.27 & 3.22 & 5.59 & 7.28 & 1.06 & ---- & ----- & ----- & ----- \\
\hline 2 & 1.91 & 2.30 & 3.19 & 4.66 & 6.52 & 1.44 & 1.61 & 2.46 & 4.71 & 5.36 & 1.01 & 1.09 & 1.91 & 3.91 & 5.20 \\
\hline 3 & 1.44 & 2.18 & 2.41 & 3.37 & 5.03 & 1.45 & 2.18 & 1.90 & 5.58 & 7.05 & 1.00 & 1.77 & 2.78 & 4.59 & 4.75 \\
\hline 4 & 1.18 & 2.19 & 3.22 & 5.97 & 7.99 & 1.82 & 2.08 & 4.08 & 5.31 & ----- & 1.61 & 2.63 & 4.187 & 8.12 & 10.60 \\
\hline 5 & 1.12 & & 2.68 & 4.12 & 6.06 & 1.46 & 2.16 & 3.10 & 4.34 & 7.41 & 1.46 & 1.39 & 1.54 & 3.25 & 2.89 \\
\hline Avg. & $\begin{array}{c}1.23 \\
\pm 0.07\end{array}$ & $\begin{array}{r}1.80 \\
+0.11\end{array}$ & $\begin{array}{l}3.0 \\
\pm 0.14\end{array}$ & $\begin{array}{c}4.73 \\
+0.36\end{array}$ & $\begin{array}{c}6.73 \\
\pm 0.45\end{array}$ & $\begin{array}{r}1.53 \\
\pm 0.05\end{array}$ & $\begin{array}{c}2.06 \\
\pm 0.67\end{array}$ & $\begin{array}{r}2.55 \\
\pm 0.35\end{array}$ & $\begin{array}{l}5.106 \\
\pm 0.52\end{array}$ & $\begin{array}{c}5.42 \\
+0.92\end{array}$ & $\begin{array}{c}1.22 \\
\pm 0.07\end{array}$ & $\begin{array}{c}1.37 \\
\pm 0.15\end{array}$ & $\begin{array}{c}2.08 \\
\pm 0.23\end{array}$ & $\begin{array}{c}4.97 \\
+0.46\end{array}$ & $\begin{array}{c}4.68^{\star} \\
\pm 0.76\end{array}$ \\
\hline
\end{tabular}

Student's t test: *Significant difference $(p<0.05)^{\star *}$ highly significant difference $(p<0.01)$

Table 3 (b): Growth index of pups of all three groups.

postnatal phase was found to decrease in a dose- related manner. The viability was affected both by natural death or cannibalism (Figure 4).

The reason for this may be explained on the basis of lactation that the harmful compounds present in the dose concentration of the leachate were excreted in milk without being detoxified and thus exerted their influence and proved lethal to some of them. This is substantiated by the fact the toxic effects on the suckling infants feeding only on mother's milk most prenatal insults manifested themselves postnatally as also reduced viability and impaired growth [9].

Large number of neonatal deaths may also be attributed to diminished secretory function of the mammary gland [10]. It could also be possible that the chemical could be excreted in the milk and render it unpalatable to the infant, which would then be unable to survive due to lack of essential nutrients, which otherwise it might be getting from its mother for its survival [11].

Poisoning of the newborn via chemicals present in the breast milk is an avenue for neonatal toxicity for those compounds that cannot cross the placenta and for continued exposure and toxicity extending into the postpartum period and later stages of development. Pollutants in breast milk are generally characterized by high lipid solubility, resistance to degradation, and slow excretion rates. Long term low level exposure results in accumulation and lactation is the only way by which large amount of the chemicals can be excreted.

Sludge leachate administered to pregnant females during growth phase, lactation and weaning were found to affect the growth of the neonates in all the treated groups. It may be attributed to poor maternal behavior by the dams that appeared to pay less attention to their offspring possibly due to chemical toxicity leading to maternal stress and lethargy or due to poor lactation thus affecting the fetal growth, leading to reduced body weight.

Since due to toxicity of the chemicals present in sludge leachate, many facial tumors and defects in limbs were noticed in dams treated during this phase, it may also be one of the reasons of mother's illness and mortality that the growth and weaning index was found to be low 


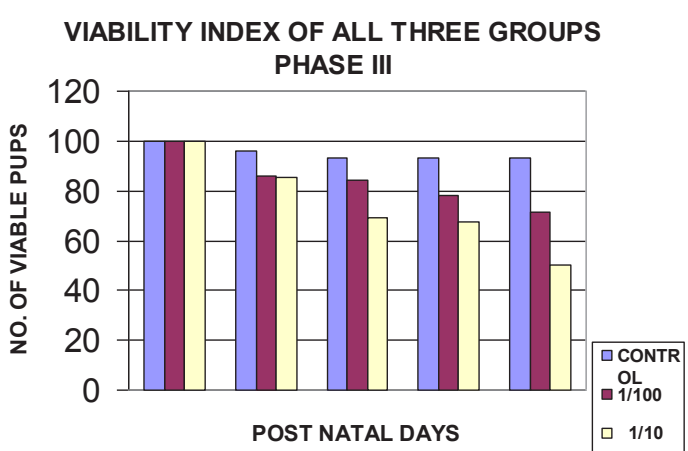

Figure 4: Viability index of all three groups phase III

in all groups. According to Schwartz et al. [12] and Mooty et al. [13] it has been suggested that low level lead exposure may impair growth in children.

Bagnell and Ellenberger [14] reported that tetrachloroethylene, a solvent present in dry-cleaning fluid, has been reported to cause cholestatic jaundice in a breast fed infant. The mother was exposed to vapours chronically, which accumulated in her breast milk. This chlorinated hydrocarbon is therefore fetotoxic. An extensive list of solvents shown to be fetotoxic in various animal systems was tabulated by Wilson [15].

Hence, the present study has clearly brought out that the sludge from the textile industry, which is dumped in open landfills, and with the potential of mixing with ground and surface water, may pose serious threat to human babies and fetuses and other mammalian fauna of the area. Thus our investigation is a step towards evaluating such adverse effects on the teratological, embryotoxicological and reproductive aspects and it is evident by the results obtained that they could serve as baseline data for the toxic manifestations of pollution caused by dyeing industry.

\section{Summary}

The leachate when administered during gestational stages reduced significantly the weight gain of dams, but was not maternotoxic since along with reduction in weight no maternal mortality was reported.

Fetotoxicity, as evidenced by the increased occurrence of several skeletal variants and decreased fetal body weights, increased number of resorbed, dead and stunted fetuses, significantly decreased placental weighs were noted at the higher dose level given during pre-implantation period, with both dose levels when leachate was administered $15^{\text {th }}$ day of gestation, lactation and weaning.

The occurrence of both visceral and skeletal variations indicates towards the potential of leachate to cause dysmorphogenic effects.

Reduced weaning index and weighs of neonates due to leachate treatment during growth phase suggests that leachate affects neonatal growth and survival.

\section{Acknowledgement}

The authors are very grateful to the Poddar International College, Mansarovar, Jaipur. We are also Thankful to Rajasthan University for encouragement and providing laboratory facilities for carrying out this work.

\section{References}

1. Gopal B, Wetzel RG (1995) Limnology in developing countries. International
Scientific Publications for the International Association of Theoretical and Applied Limnology (SIL) 1: 230.

2. Chhonkar PK, Datta SP, Joshi HC, Pathak H (2000) Impact of Industria effluents on soil health and agriculture - Indian experience: part- II - tannery and textile industrial effluents. J Sci Ind Res 59: 446-454.

3. Mohnot SM, Dugar S (1987) Textile industry and water pollution problem of Western Rajasthan. In environmental degradation of Western Rajasthan (Ed. Mohnot. S.M. and Bhandari. M.M) 63-71.

4. Mohnot SM, Durve VS (1989) Evaluation of the Hazards of Untreated and Treated textile dyeing and printing waste on mammals. Technical Report VIII=61. Man and Biosphere Programme. Govt. of India. New Delhi.

5. Soni P, Sharma S, Sharma S, Kumar S, Sharma KP (2006) A comparative study on the toxic effects of textile dye wastewaters (untreated and treated) on mortality and RBC of a freshwater fish Gambusia affinis (Baird and Gerard). $J$ Environ Biol 27: 623-628.

6. Luo D, Das S, Vincent SR (1995) Effects of methylene blue and LY83583 on neuronal nitric oxide synthase and NADPH-diaphorase. Eur J Pharmacol 290: 247-251.

7. Olshan AF, Faustman EM (1993) Male medicated developmental toxicity. Annu Rev Publ Health 14: 159-181.

8. Sokal RR, Rohlf FJ (1973) Introduction to Biostatistics. WH Freeman \& Co. Ltd., 17: 466-467.

9. Kavlock RJ, Short RD Jr, Chernoff N (1987) Further evaluation of an in vivo teratology screen. Teratog Carcinog Mutagen 7: 7-16.

10. Barnes JM, Denz FA (1951) The chronic toxicity of p-nitrophenyl diethy thiophosphate (E. 605); a long-term feeding experiment with rats. J Hyg (Lond) 49: 430-441.

11. FDA (1970) National Agriculture Chemical Associations, NAC, News pesticides Review 28.

12. Schwartz J, Angle C, Pitcher H (1986) Relationship between childhood blood lead levels and stature. Pediatrics 77: 281-288.

13. Mooty J, Ferrand CF, Harris P (1975) Relationship of diet to lead poisoning in children. Pediatrics 55: 636-639.

14. Bagnell PC, Ellenberger HA (1977) Obstructive jaundice due to a chlorinated hydrocarbon in breast milk. CMAJ 117: 1047.

15. Wilson JG (1977) Teratogenic effects of environmental chemicals. Fed Proc 36: 1698-1703. 